\title{
Entwicklungskonzept Alpenrhein
}

\author{
Development concept river alpine rhine
}

\author{
von B. Zarn
}

\section{Kurzfassung/Summary}

Mit den in den letzten $150 \mathrm{Jah}$ ren ausgeführten Korrektionen konnte die Abflusskapazität im Alpenrhein auf den gewünschten Ausbaustandard erhöht werden. Der Geschiebehaushalt und die Morphologie des Alpenrheins wurden mit den Korrektionen aber erheblich verändert. Trotzdem ist der Geschiebehaushalt nicht im Gleichgewicht. Die Maßsnahmen führten $\mathrm{zu}$ einer deutlichen Abnahme der Strukturvielfalt und der Lebensräume, was sich nachteilig auf die Ökologie auswirkt. Damit verbunden ist auch ein Rückgang der Attraktivität als Naherholungsgebiet. Aufgrund der Wechselwirkung mit dem Grundwasser ist das ganze Rheintal betroffen. Auch die Wasserkraftnutzung im Einzugsgebiet des Alpenrheins wirkt sich wegen des Schwalls negativ auf die Gewässerökologie aus. Im Entwicklungskonzept Alpenrhein werden Maßnahmen zur Reduktion der Defizite vorgeschlagen. Im Zentrum steht mehr Raum für den Alpenrhein. So sollen Flussaufweitungen oberhalb von Buchs gegen die fortschreitende Eintiefung wirken und oberhalb des Bodensees die Abflusskapazität erhöhen, wo das Schadenpotenzial besonders hoch ist. Die Flussaufweitungen beeinflussen das Grundwasser positiv, erhöhen die morphologische und ökologische Vielfalt und schaffen wertvolle Erholungsräume im Rheintal. Weiter werden ein Gewässerraum und ein Konzept für die Bewältigung von Hochwasserereignissen, welche größer als der Ausbaustan- dard sind, vorgeschlagen.

Key words: Alpenrhein, Entwicklungskonzept, Hochwasserschutz, Geschiebehaushalt, Grundwasser, Gewässerökologie

The discharge capacity of the Alpine Rhine River could have increased on the desired standard due to the systematic river training of the past 150 years. However, this caused a considerable change in the bed load sediment budget and the morphology. In spite of that, the bed load sediment budget is not in equilibrium. The flood protection measures led to a severe reduction of natural sound habitats, which affects adversely the ecology. With it dropped the attractiveness of the Alpine Rhine as a recreation area. Because of the interaction with the ground water table the whole Rhine Valley is affected. The usage of the hydro power in the catchment of the Alpine Rhine River affects the river ecology negatively due to the daily discharge surges. In the Development Concept Alpine Rhine several measures are proposed to reduce the deficits. Overall, more space is needed for the Alpine Rhine. The river bed enlargements proposed upstream of Buchs function against the continuous erosion and the ones proposed upstream of the Lake of Constance increase the discharge capacity, where the damage potential is extra high. The river bed enlargements affect the ground water favourably, increase the morphological and ecological diversity and create valuable recreation areas. In addition a river space (to avoid settlement and infrastructure directly behind the river bank) and a concept to cope with flood events larger than the design discharge are proposed.

Key words: River Alpine Rhine, development concept, flood protection, bed load sediment budget, groundwater, river ecology

\section{Einleitung}

In der Vergangenheit standen beim Alpenrhein Sicherheitsinteressen im Vordergrund. Die Maßnahmen zielten darauf ab, die Hochwässer sicher abzuleiten. Dies war die Voraussetzung, um das Rheintal besiedeln und bewirtschaften zu können. Mit der Entwicklung stieg der Bedarf an Ressourcen. Der Alpenrhein lieferte Kies, elektrische Energie und Trinkwasser. Die Auswirkungen auf den Feststoffhaushalt oder die Ökologie wurden erst nach langen Zeiträumen ersichtlich. Der Verlust von natürlichen Lebensräumen ist für Pflanzen und Tiere, aber auch für den Menschen mit seinem Bedürfnis nach Erholung und Freizeitgestaltung von Bedeutung.

Der Alpenrhein muss heute und in Zukunft verschiedene Aufgaben und Funktionen erfüllen. Weil dieser Gebirgsfluss weder räumlich isoliert noch thematisch sektoral betrachtet werden kann, haben sich die Kantone Graubünden, St. Gallen sowie das Fürstentum Liechtenstein und das Land Vorarlberg gemeinsam mit Österreich und der Schweiz entschlossen, ein Entwicklungskonzept für den Alpenrhein von Reichenau bis zum Bodensee zu erarbeiten. Das Schwergewicht der Untersuchung wurde auf den Alpenrhein als Gewässer und das Thema Leben und Arbeiten mit engem Be- 


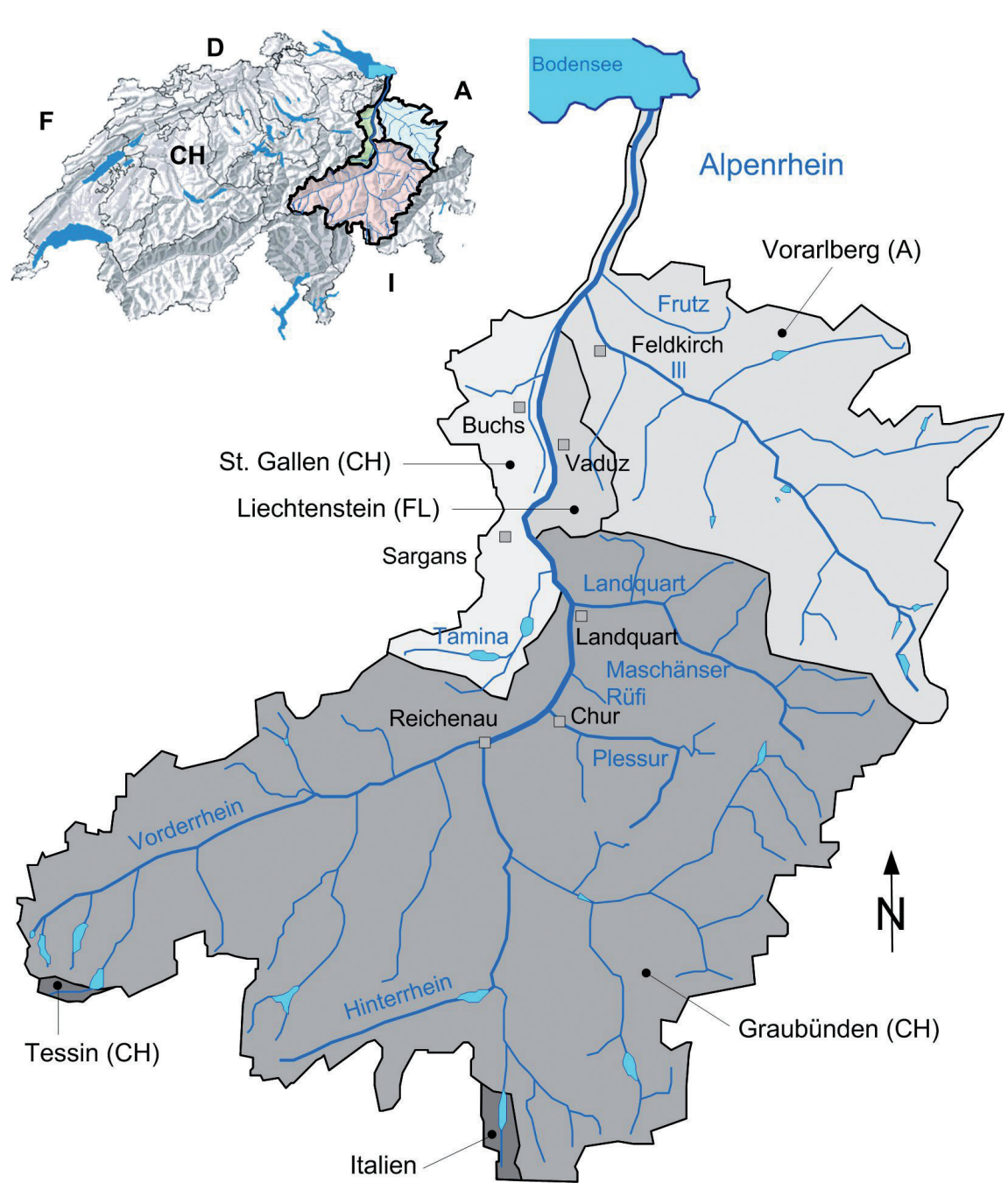

Abb. 1: Einzugsgebiet des Alpenrheins und seiner Zuflüsse.

zug zum Alpenrhein gelegt. Die Hauptthemen der Untersuchung waren Hochwasserschutz, Ökologie, Grundwasser sowie Leben und Arbeiten. An der Erarbeitung des Entwicklungskonzepts waren Fachpersonen aus verschiedenen Disziplinen beteiligt. Es basiert auf den Resultaten von zahlreichen Einzelstudien verschiedener Fachrichtungen. Die wichtigen Grundlagen sind im Literaturverzeichnis aufgeführt. Im ersten Teil des Beitrages wird der Alpenrhein beschrieben und im zweiten Teil werden die wichtigsten Vorschläge des Entwicklungskonzeptes zusammengefasst.

\section{Der Alpenrhein heute}

\subsection{Einzugsgebiet}

Bei Reichenau vereinigen sich die beiden etwa gleich großen Flüsse Vorder- und Hinterrhein und bilden den Alpenrhein (Abb. 1 und Abb.2). Er mündet nach rund $90 \mathrm{~km}$ in den Bodensee und entwässert ein Einzugsgebiet von $6123 \mathrm{~km}^{2}$, welches in der Schweiz (Kantone Graubünden, St. Gallen und Tessin), in Österreich (Land Vorarlberg), im Fürstentum Liechtenstein und in Italien liegt. Der Gletscheranteil beträgt heute weniger als $1.4 \%$ und die mittle- re Einzugsgebietshöhe liegt bei $1800 \mathrm{~m}$ ü.M. Die aus Sicht Morphologie und Feststofftransport wichtigsten Zuflüsse sind - außer dem Vorder- und dem Hinterrhein - die Plessur, die Landquart und die Ill. Neben diesen Gebirgsflüssen münden verschiedene Wildbäche wie die Maschänser Rüfi, die Tamina oder die Frutz in den Alpenrhein.

\subsection{Abflussregime}

Das Abflussgeschehen des Alpenrheins wird durch Schneeschmelze, Hochwasser und die Wasserkraftnutzung geprägt. Die Schneeschmelze führt zu hohen Sommerabflüssen mit einem ausgeprägten Abflussmaxima im Juni (Abb. 3). Die großen Speicherseen, welche ab 1950 im Einzugsgebiet des Alpenrheins erstellt wurden, verlagern einen Teil der Sommerabflüsse in den Winter. Der natürliche Abfluss wird durch tägliche Abflussschwankungen überlagert, die mit der bedarfsgerechten Energieproduktion zusammenhängt. Bei der Mündung in den Bodensee liegt der mittlere Jahresabfluss bei $230 \mathrm{~m}^{3} / \mathrm{s}$. Das 100-jährliche Hochwasser beträgt in diesem Alpenrheinabschnitt $3^{\prime} 100 \pm 200 \mathrm{~m}^{3} / \mathrm{s}$.

\subsection{Verbauungsgeschichte}

Die Morphologie des Alpenrheins ist sehr stark von den umfassenden Korrektionen geprägt, mit welchen ab Mitte des 19. Jahrhunderts begonnen wurde (Bergmeister und Kalt, 1992). Ziel der Verbauungsmaßnahmen war ein stabiles Flussbett mit einer ausreichenden Abflusskapazität. Dazu wurde das Flussbett eingeengt und mit hohen Dämmen versehen. Zudem wurde der Alpenrhein mit der Verlegung der Mündung bei Fussach (Fussacher Durchstich, 1895-1900) und der Abtrennung des Mäanders bei 

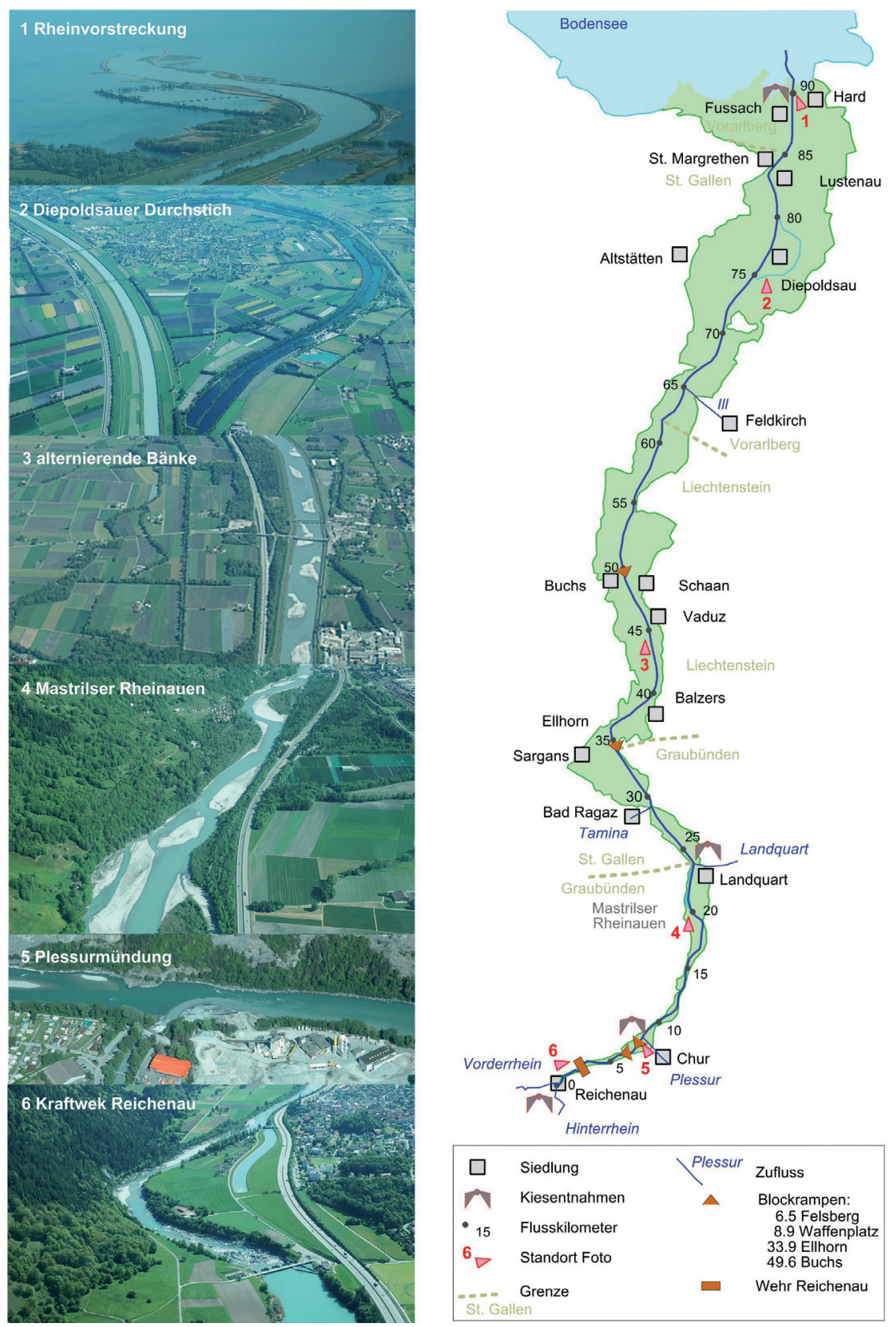

\subsection{Morphologie}

Die Geometrie des Alpenrheins ist unterschiedlich. Von Reichenau ( $\mathrm{km} \mathrm{0)}$ bis fast zur Illmündung (km 65) fließt er mit Ausnahme der Mastrilser Rheinauen (km 20-23) in einem Trapezprofil mit Bettbreiten zwischen 60 und $100 \mathrm{~m}$ (siehe auch Abb. 2). Auf der folgenden Strecke bis zum Bodensee besteht der Abflussquerschnitt aus einem 40 bis $70 \mathrm{~m}$ breiten Mittelgerinne mit überströmbaren Mittelwuhren und beidseitigen Vorländern. Der Abstand der Hochwasserschutzdämme schwankt zwischen 280 und $400 \mathrm{~m}$. In Abhängigkeit dieser unterschiedlichen Breiten ist das Flussbett verschieden ausgebildet. Oberhalb der Landquartmündung und unterhalb der Illmündung ist es mehrheitlich eben. Zwischen diesen beiden Mündungen dominieren alternierende Bänke. Einzig in den Mastrilser Rheinauen (km 20-23) hat der Alpenrhein dank einer Bettbreite von 200 bis $300 \mathrm{~m}$ noch seinen ursprünglichen Charakter und kann sich ein Teilgerinne verzweigen. Das Gefälle nimmt von Reichenau bis zum Bodensee von $0.35 \%$ auf $0.1 \% \mathrm{ab}$.

\subsection{Veränderung der Sohlenlage Seit 1940 und Entwicklung}

Wegen den Korrektionen und Kiesentnahmen tiefte sich das Flussbett im 20. Jahrhundert mehrheitlich ein, aber nicht gleichmäßig. Zwischen 1940/41 und 1950 (Differenz Nulllinie/Strich-Linie in Abb. 5) und zwischen 1974 und

Diepoldsau (Diepoldsauer Durchstich, 1908 -1923) um fast $10 \mathrm{~km}$ verkürzt. Im oberen Teil des Alpenrheins wurden in der zweiten Hälfte des 19. Jahrhunderts vier Blockrampen zur Begrenzung der Sohleintiefung gebaut. Die Ablagerung von jährlich rund 3 Mio. $\mathrm{m}^{3}$ Schwebstoffen im Bodensee führt zu einem raschen Deltawachstum (Abb. 4). Mit der sich noch im Bau befindenden Rheinvorstreckung (Verlängerung des Flusslaufs in den Bodensee, Abb. 2 oben links) werden die Schwebstoffe in tiefe Seebereiche geleitet. Damit werden die Verlandung respektive die Abtrennung des östlichsten Teils des Bodensees und Anlandungen im Alpenrhein und damit eine Abnahme der Abflusskapazität hinaus gezögert. 1995 (Differenz Punkt-Linie/ausgezogene Linie) waren die Sohlenveränderungen deutlich kleiner als in der Periode von 1950 bis 1974 (Differenz Strich-Linie/ausgezogene Linie). Die Hauptursache für die massiven Eintiefungen zwischen 1950 und 1974 waren die Kiesentnahmen. Zwar wurde seit 1936 Kies aus dem Alpenrhein gewonnen. Aber wie Abb. 5 und 


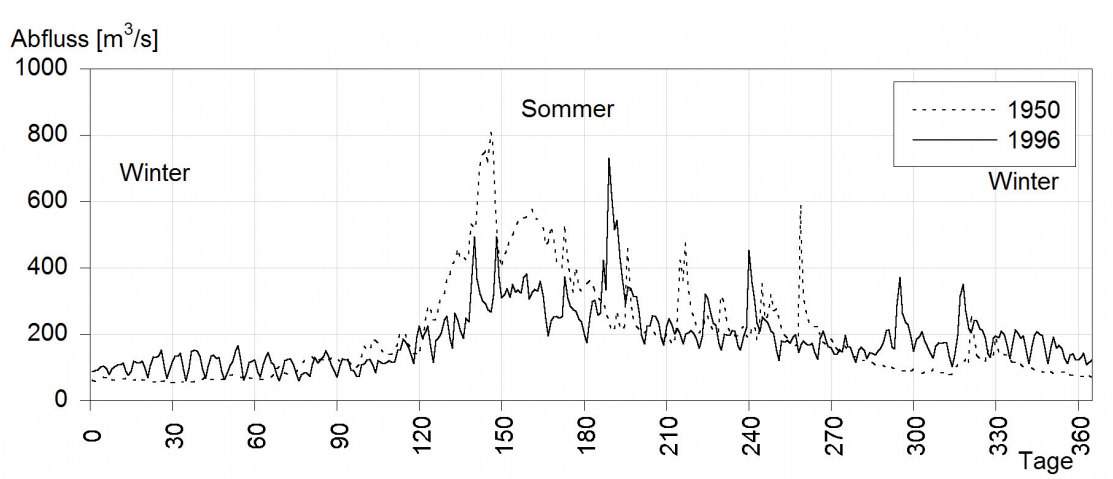

Abb. 3: Abflussganglinie bei Diepoldsau vor (1950) und nach Kraftwerksbau (1996).

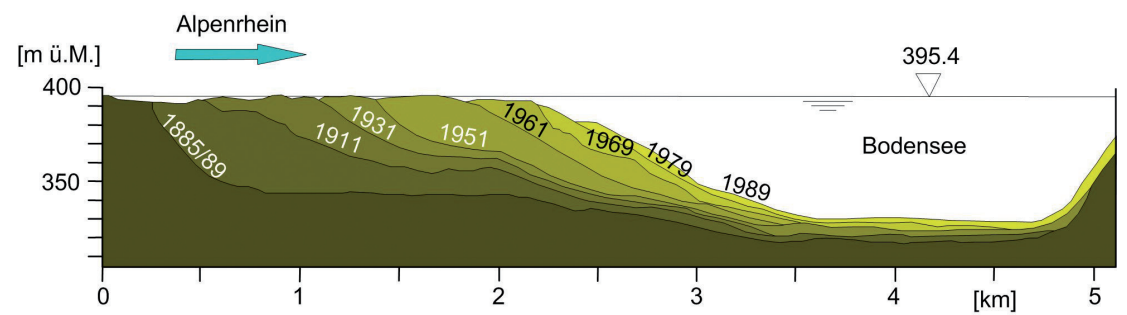

Abb. 4: Längenprofil des Alpenrheindeltas im Bodensee seit 1885 (aus Lambert 1989)

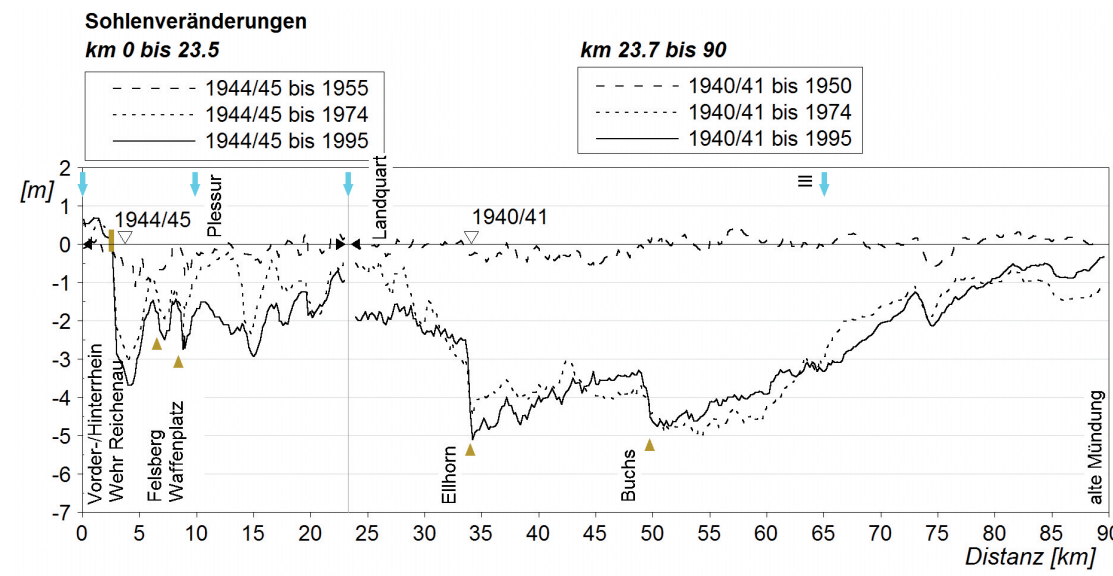

Abb. 5: Sohlenveränderungen im Alpenrhein zwischen 1941 und 1995.

Abb. 6 zeigen, fallen die Hauptentnahmemengen in die Periode mit den größten Eintiefungsraten zusammen, vor allem in der Strecke zwischen den Mündungen der Landquart und der Ill ( $\mathrm{km} 23.7$ bis $\mathrm{km}$ 65). Nach dem Einsturz der Brücke Buchs-Schaan im Jahr 1972 wurden in diesem Bereich die Kiesentnahmen stark eingeschränkt.

Zwischen 1936 und 1990 wur- den insgesamt 29 Mio. $\mathrm{m}^{3}$ Geschiebe entnommen (Abb. 6), was rund fünf Mal mehr ist als die heutigen Geschiebeeinträge aller Zuflüsse in einer vergleichbaren Zeitspanne. Es erstaunt deshalb nicht, dass sich beispielsweise das Flussbett beim Ellhorn (km 33.9) oder bei Buchs (km 50) zwischen 1950 und 1974 um rund $5 \mathrm{~m}$ eintiefte (Abb. 5, Differenz Strich-Linie zu Punkt-Linie).

\subsection{Grundwasser und Trink- wasser}

Wie im Beispiel von Abb. 7 führte die Absenkung des Flussbettes um $5 \mathrm{~m}$ zwischen 1953 und 1973 auf längeren Abschnitten des Alpenrheins dazu, dass Rheinwasser nicht mehr in das Grundwasser infiltriert, sondern Grundwasser in den Alpenrhein fließt. Mit der Einstellung der Kiesentnahmen nach 1972 führten Anlandungen von rund $1 \mathrm{~m}$ bis 1998 in Rheinnähe $\mathrm{zu}$ etwas höheren Grundwasserspiegeln.

Das Grundwasser ist die wichtigste Ressource für die Trinkwasserversorgung. Aus ihm wird mehr als die Hälfte des Trinkwassers für die 450.000 BewohnerInnen des Rheintales gewonnen. Qualität und Ergiebigkeit sind wegen der Sohleintiefung in den letzten Jahrzehnten geringer geworden. Das Absinken des Grundwasserspiegels hat wertvolle Lebensräume in grundwassergespeisten Binnengewässer (Giessen) und in Feuchtgebieten beeinträchtigt. Gleichzeitig ist die Vernässung von Landwirtschaftsflächen zurückgegangen, was die Bewirtschaftung begünstigt.

\subsection{Unterschiedliche Abfluss- kapazität}

Die zum Teil massive Eintiefung des Rheins in den letzten Jahrzehnten bewirkte vor allem zwischen Sargans und der Illmündung eine sehr grosse Abflusskapazität. In diesem Abschnitt können Hochwassermengen abgeführt werden, welche weit über dem üblichen Ausbaustandard (HQ100) liegen. Auch im Kanton Graubünden ist die Abflusskapazität in der Regel höher als das HQ100. In der internationalen Rheinstrecke zwischen der Illmündung und dem Bodensee ist die Abflusskapazität für das HQ100 gerade noch gewährleistet. 


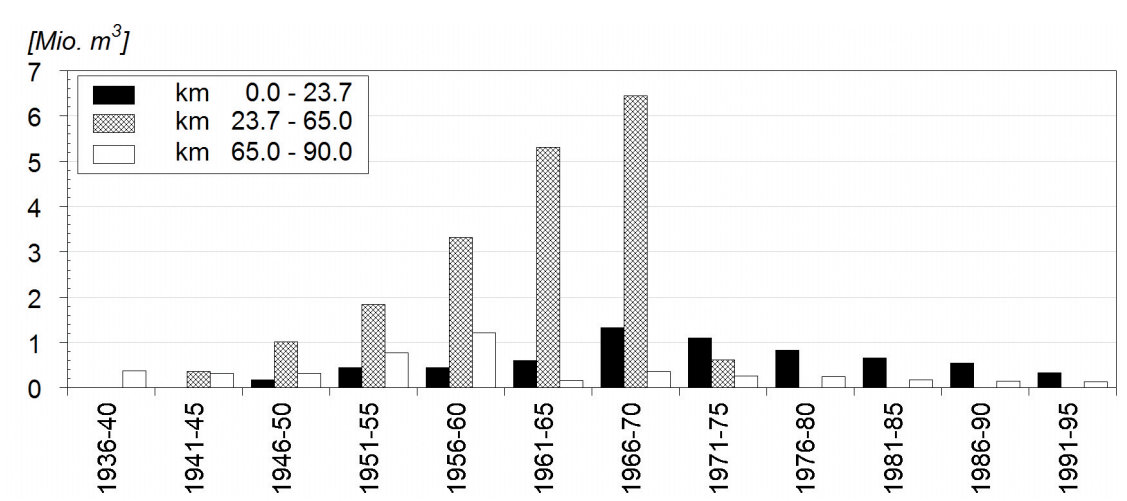

Abb. 6: Geschiebeentnahmen im Alpenrhein in verschiedenen Teilstrecken seit 1936.

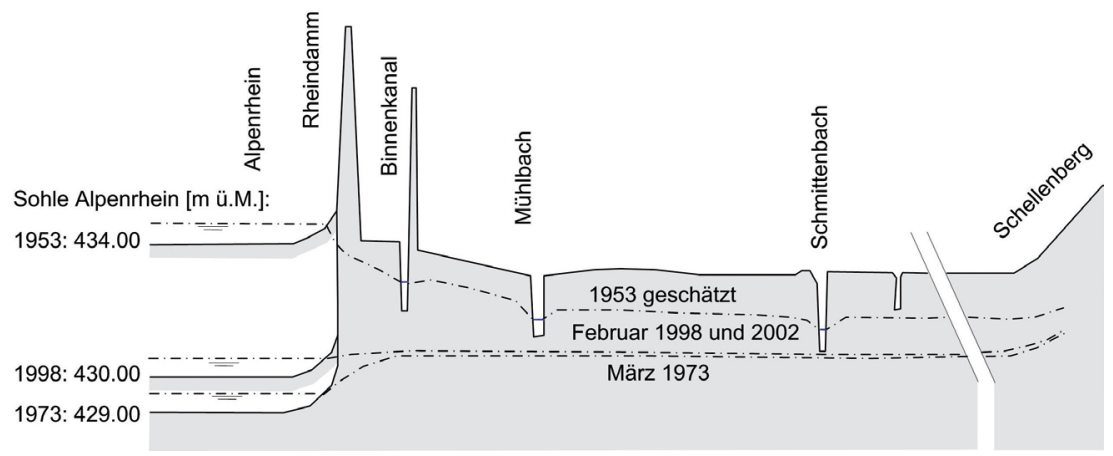

Abb. 7: Einfluss der Rheinsohle auf den Grundwasserspiegel am Beispiel von Ruggell im Fürstentum Liechtenstein (Rheinkilometer 58.4, schematische und überhöhte Darstellung).

\subsection{Hochwasserschutz und Entwicklung Rheintal}

Der gute Hochwasserschutz war eine wichtige Voraussetzung für die rasante Entwicklung des Rheintals. Vor der systematischen Rheinkorrektion konzentrierten sich die Siedlungen mit wenigen Ausnahmen auf die zahlreichen Wildbachkegel, weil der Alpenrhein regelmässig die Talebene überschwemmte. Dank der umfangreichen Schutzmaßnahmen am Alpenrhein blieb das Rheintal seit 1927, als bei Schaan im Fürstentum Liechtenstein (Abb. 2) der Hochwasserschutzdamm zum letzten Mal brach, vor Überschwemmungen des Alpenrheins mehrheitlich verschont. Dies führte dazu, dass sich die Siedlungen zunehmend in der Talebene entwickelt haben (Abb.8). Die Bevölkerung stieg zwischen 1960 und 2000 von 280.000 auf 451.000 EinwohnerInnen. Die Anzahl der Arbeitsplätze nahm zwischen 1970 und 2000 von 150.000 auf 238.000 zu. Mit dieser Entwicklung erhöhte sich auch das Schadenpotenzial vor allem im mittleren und unteren Rheintal. Bei einem Dammbruch muss heute im unteren Rheintal mit Schadenssummen von bis $\mathrm{zu}$ mehreren Milliarden Euro gerechnet werden.

\section{9 Ökologie}

Heute ist der Alpenrhein fast durchgehend reguliert und durch Hochwasserschutzdämme von seinen Nebengewässern und $\mathrm{Au}$ wäldern abgetrennt. Die Zuflüsse sind im Mittel- und Unterlauf zu Binnenkanälen zusammengefasst. Die wenigen verbliebenen Mündungen sind wegen der Sohleintiefung des Alpenrheins mehr- heitlich für Fische unpassierbar. Die Zuflüsse sind durch menschliche Eingriffe wesentlich beeinträchtigt. Tägliche Wasserspiegelschwankungen im Meterbereich durch die Wasserkraftnutzung beeinträchtigen zusätzlich die Funktionsfähigkeit der verbliebenen aquatischen Lebensräume und den Erholungswert des Flusses. Der Verlust der Gewässervielfalt führte zum Aussterben vieler Tierund Pflanzenarten und zu Bestandesreduzierungen bei den verbliebenen Arten.

\subsection{Entwicklung Geschiebe- haushalt}

Prognoserechnungen über den Geschiebehaushalt zeigen, dass bei gleichen Voraussetzungen wie zwischen 1974 und 1995 oberhalb von Buchs mehrheitlich Eintiefungen und unterhalb davon Anlandungen $\mathrm{zu}$ erwarten sind (Abb. 9). Allerdings nehmen die Sohlenveränderungen pro Jahr und gegenüber den früheren $\mathrm{Pe}$ rioden etwas $a b$, weil durch die Veränderung des Gefälles infolge von Ablagerungen und Erosionen das Transportvermögen ausgeglichener wird. Weil insgesamt geringere Erosionen prognostiziert werden, kann weniger Geschiebe mobilisiert und transportiert werden als in der Eichperiode von 1974

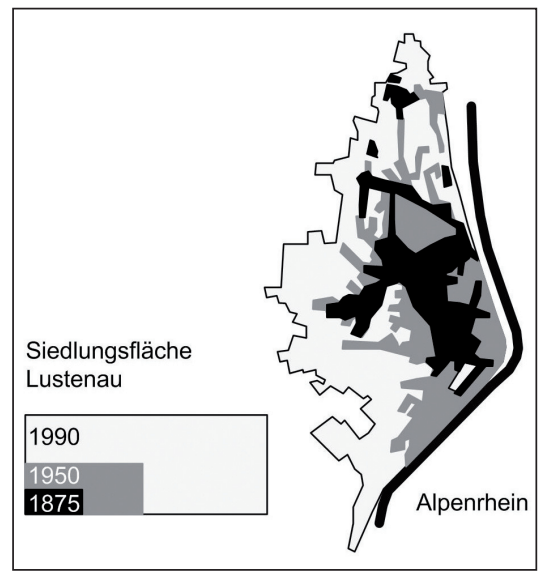

Abb. 8: Entwicklung der Siedlungsfläche von Lustenau (Land Vorarlberg) seit 1875. 

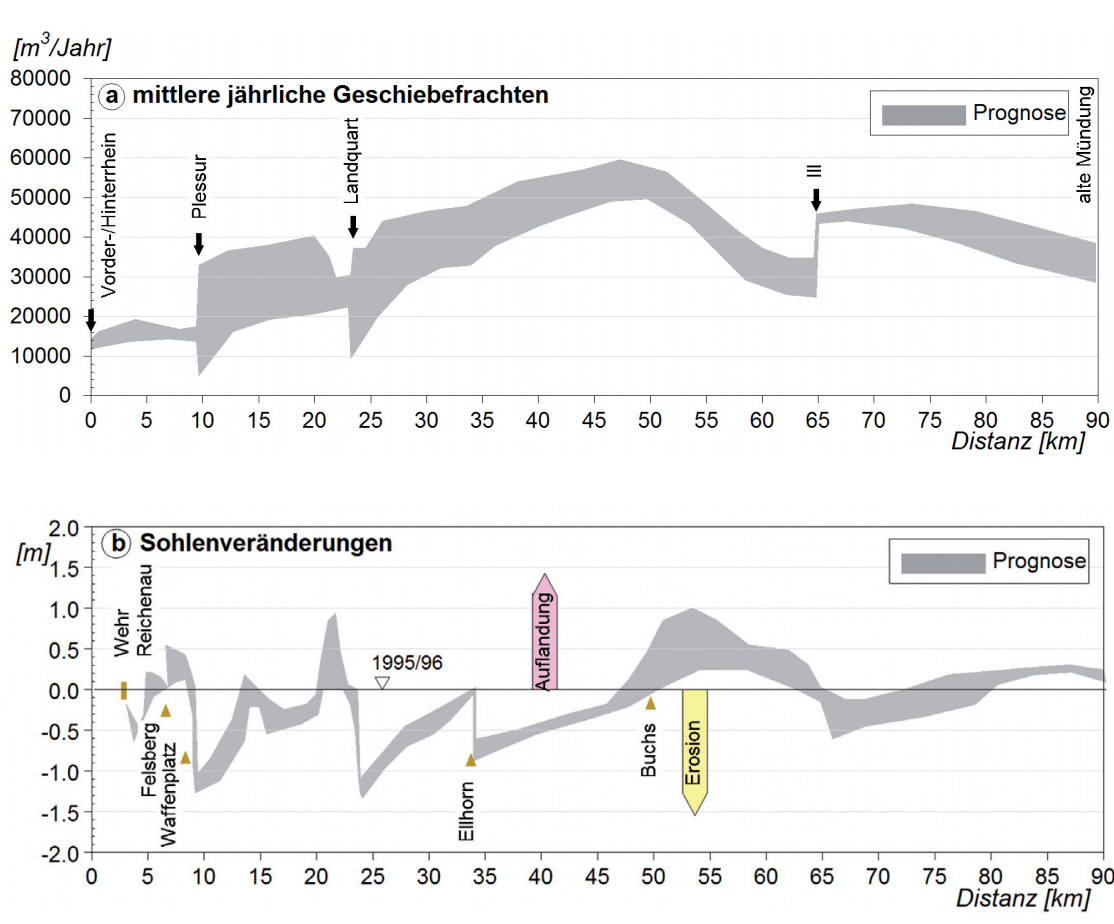

Abb. 9: Prognose der mittleren jährlichen Geschiebefrachten (a) und Sohlenveränderungen (b) in den nächsten 25 Jahren für Bedingungen wie in der Periode von 1974 bis 1996.

bis 1996. Zum Beispiel wurden unterhalb der Landquartmündung zwischen 1974 und 1996 lokal Erosionen von insgesamt über $1.5 \mathrm{~m}$ beobachtet. Für eine gleich lange Periode in der Zukunft werden „nur“ noch Erosionen von rund $1.2 \mathrm{~m}$ prognostiziert. Treten in den nächsten Jahren eher größere Abflüsse als zwischen 1974 und 1996 auf, ist mit einer Beschleunigung der Prozesse zu rechnen, im Fall von kleineren Abflüssen mit einer Verzögerung. Aus Abb. 9 oben geht hervor, dass der Alpenrhein Geschiebefrachten in der Größenordnung von $10^{\prime} 000$ bis $60^{\prime} 000 \mathrm{~m}^{3} /$ Jahr transportiert.

\section{Entwicklungskonzept}

\subsection{Handlungsbedarf}

Damit auch in Zukunft das Rheintal mit seinem hohen Schadenpotenzial vor Hochwasser angemessen geschützt wird und der Alpenrhein seine vielfältigen Funktionen als Lebensader erfüllen kann, braucht dieser Gebirgsfluss mehr Raum für:

- die langfristige Erhöhung der Abflusskapazitätzwischen Diepoldsau und Bodensee wegen dem hohen Schadenpotenzial

- die Reduktion des Geschiebetransportvermögens vor allem in den langen Eintiefungsstrecken oberhalb von Buchs

- die Schadensminderung bei extrem seltenen Hochwasserereignissen, welche größer als die Ausbauwassermenge sind

- die Erhöhung der morphologischen und ökologischen Vielfalt

- die Lösung der Schwallproblematik, welche durch die bedarfsorientierte Energieproduktion der Wasserkraftanlagen im Einzugsgebiet verursacht wird.

- die Wiederherstellung der Passierbarkeit für Fische im Alpenrhein selbst und in die Zuflüsse

- die Erhaltung des Potenzials für die Grundwasser- und Wasserkraftnutzung

\subsection{Flussaufweitungen}

Eine wichtige Maßnahme im Entwicklungskonzept sind Flussaufweitungen (Abb. 10). Oberhalb von Buchs wirken sie gegen die fortschreitende Eintiefung und im Abschnitt oberhalb des Bodensees, wo das Schadenpotenzial besonders hoch ist, kann die Abflusskapazität erhöht werden. Die Flussaufweitungen beeinflussen das Grundwasser positiv, erhöhen die morphologische und ökologische Vielfalt und schaffen wertvolle Erholungsräume für BewohnerInnen und Gäste des Rheintals.

\subsection{Gewässerraum}

Die für die Umsetzung der im Entwicklungskonzept vorgeschlagenen Maßnahmen beanspruchen Raum, welcher mit Hilfe der Raumplanung freigehalten werden soll. Dazu wird ein Gewässerraum vorgeschlagen, welcher den Bereich zwischen den Dämmen und rheinnahe Gebiete umfasst. Mit dem Gewässerraum soll der erforderliche Handlungsspielraum für Maßnahmen zu Gunsten Hochwasserschutz, Grundwasser, Gewässerökologie, Wasserkraftnutzung und Naherholung erhalten werden. Dieser Handlungsspielraum ist wichtig, weil die Umsetzung des Entwicklungskonzeptes viel Zeit beanspruchen wird und sich die Bedürfnisse oder die Ausgangslage (z.B. Zunahme der Hochwasser infolge Klimaerwärmung, Deltawachstum) ändern können.

\section{4 Überlastfallkonzept}

Verschiedene vergangene Hochwasserereignisse, wie diejenigen vom August 2005, haben gezeigt, dass bei extremen Ereignissen die Hochwasserspitzen die bisher beobachteten Abflusswerte und die darauf basierenden Ausbau- 


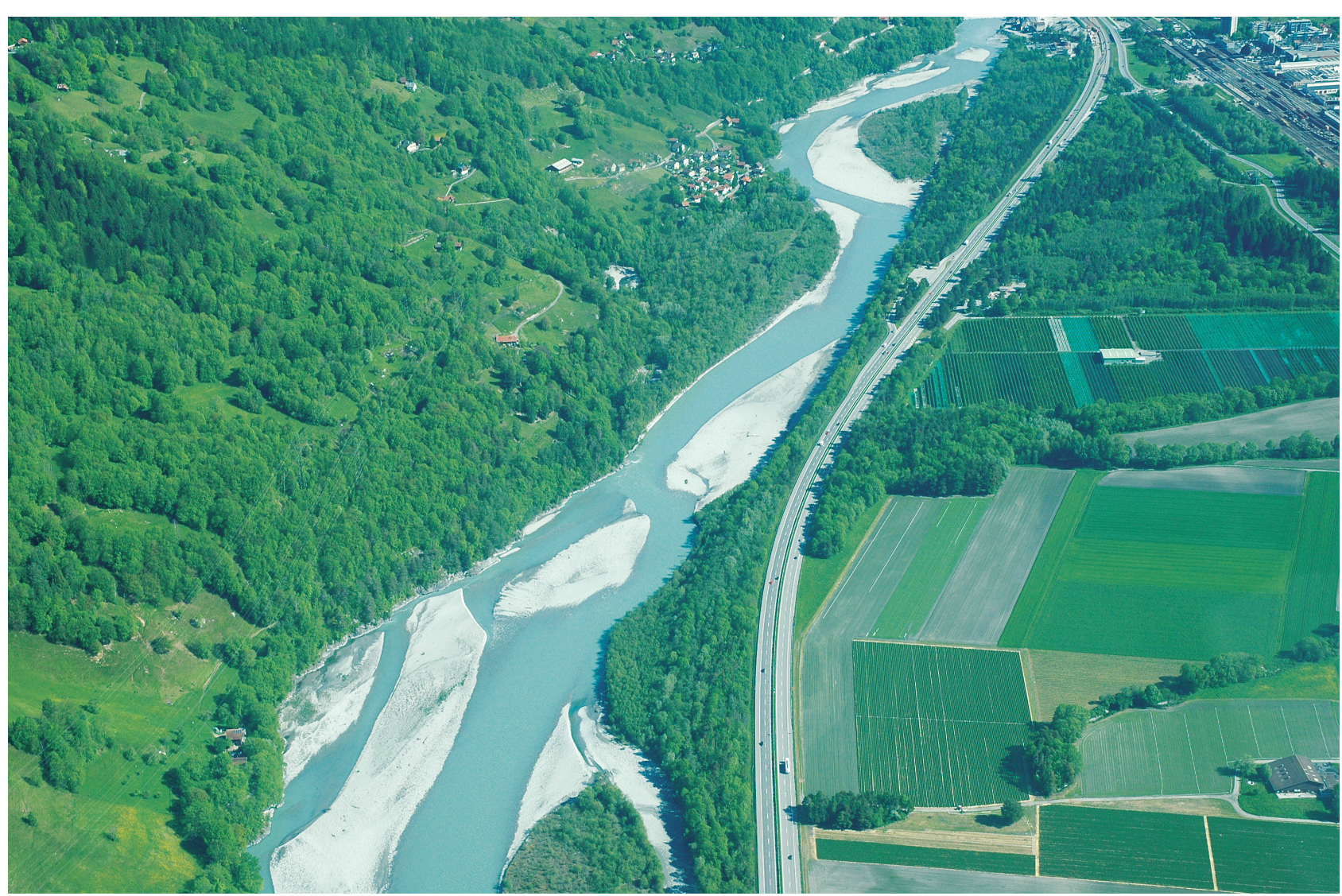

Abb. 10: Die Mastrilser Rheinauen sind eine wichtige Referenzstrecke für die Planung von Flussaufweitungen am Alpenrhein.

kapazitäten deutlich übertreffen können. Bei einem solchen Überlastfall am Alpenrhein muss heute mit zufälligen Dammbrüchen gerechnet werden. Große Wassermengen können Gebiete mit hohem und niedrigem Schadenpotenzial überfluten. Das Ent- wicklungskonzept Alpenrhein schlägt die Erarbeitung eines Überlastfallkonzepts mit vorgegebenen Notentlastungsstellen vor. Dort wird im Überlastfall nur das nicht beherrschbare Wasser in Gebiete mit niedrigem Schadenpotenzial geleitet. Zusätz- liche Leitmaßnahmen begrenzen das Überflutungsgebiet.

Korrespondenz:

Hunziker, Zarn \& Partner AG

Via Flucs 10, 7013 Domat/Ems

bzarn@hzp.ch

\section{LITERATUR}

ARGE Trübung Alpenrhein (2002): Trübung und Schwall im Alpenrhein, Kurzfassung; im Auftrage der Projektgruppe Gewässer- und Fischökologie der Intern tonalen Regierungskommission Alpenrhein.

BERGMEISTER, U.; KALT, L. (1992) „Der Alpenrhein und seine Regulierung“. (Hrsg.) Internationale Rheinregulierung, BuchsDruck und Verlag, Buchs.

Büro für Hydrogeologie Dr. P. Angehrn AG, TK Consult AG, Rudhardt+Gasser (2000) „Grundwasserhaushalt Alpenrhein. Grundwassermodellierung für den Abschnitt Landquart - Bodensee“. Im Auftrag der Internationalen Regierungskommission Alpenrhein

Eberstaller, J.; Haidvogl, G.; Jungwirth, M. (1997) „Gewässer-\&fischökologisches Konzept Alpenrhein - Grundlagen zur Revitalisierung mit Schwerpunkt Fischökologie“. Herausgeber: Internationale Regierungskommission Alpenrhein, Projektgruppe Gewässer- und Fischökologie, ISBN 3-9500562-1.

Heierli AG, Tergeso AG, Hunziker, Zarn \& Partner AG (2000) Hydogin A (2000) "Hydrologie Alpen, penrhein.
Hochschule für Technik Rapperswil, TK Consult AG, Hunziker, Zarn \& Partner AG (2004) ,Revitalisierun und Wasserkraftnutzung am Alpenrhein“. Im Auftrage der Internationalen Regierungskommission Alpenrhein (unveröffentlicht)

Hunziker, Zarn \& Partner AG (2001) „Morphologi und Geschiebehaushalt Alpenrhein, Zusammenfassender Bericht über die Untersuchungen zwischen 198 und 2000“. Im Auftrag der Projektgruppe Flussbau der Internationalen Regierungskommission Alpenrhein

IG Flussbau Alpenrhein (2003) „Schadenrisiken un Schutzmassnahmen im Alpenrheintal“. Im Auftrag de Projektgruppe Flussbau der Internationalen Regierungskommission Alpenrhein.

Internationale Regierungskommission Alpenrhei (IRKA) und Internationale Rheinregulierung (IRR) (2005) Entwicklungskonzept Alpenrhein" Kurzbich

Lambert A (1989): Das Rheindelta im See Vermessung" Photogrammetrie, Kulturtechnik, Nr. 1.
Schweizerischer Wasserwirtschaftsverband und Versuchsanstalt für Wasserbau, Hydrologie und Glaziologie der ETH Zürich (2004) „Schwallreduktion un Hochwasserspitzenminderung im Alpenrhein, Möglich Massnahmen und deren Auswirkungen". Im Auftrage de Internationalen Regierungskommission Alpenrhein.

Strittmatter Partner AG, St. Gallen (2002) „Räumliche Entwicklung des Alpenrheintals-Analysen und Thesen“. Im Auftrag der Raumplanungsfachstellen de Fürstentums Liechtenstein, des Kantons St. Gallen, des Kantons Graubünden und des Landes Vorarlberg.

Zarn, B.; Oplatka, M.; Pellandin, St.; Mikos, $M_{\text {, }}$ Hunziker, R.; Jäggi, M. (1995) „Geschiebehaushalt Alpenrhein; Neue Erkenntnisse und Prognosen über die Sohlenveränderungen und den Geschiebetransport". Mitteilung Nr. 139 der Versuchsanstalt für Wasserbau, Hydrologie und Glaziologie der ETH Zürich, Zürich, 187 Seiten. 